\title{
Estudo da postura e equilíbrio do atleta por meio de fotogrametria e estabilometria no uso de protetor bucal para esporte
}

\author{
Study of the athlete's posture and balance through photogrammetry and stabilometry when using \\ mouthguards for sports \\ Estudio de la postura y el equilibrio del deportista mediante fotogrametría y estabilometría cuando \\ se utilizan protectores bucales para la práctica deportiva
}

\section{Resumo}

Os protetores bucais são dispositivos intraorais que visam prevenir traumatismos dentários e maxilofaciais decorrentes da prática esportiva. Por ser um dispositivo interoclusal, é necessário entender e saber se este pode interferir no equilíbrio corporal do atleta, tendo em vista que a existência da relação entre o sistema temporomandibular e o controle postural já foi comprovada analisando diferentes oclusões dentarias e posições mandibulares. O objetivo deste estudo foi avaliar a influência do protetor bucal individualizado (PBI) no equilíbrio e postura corporal como também extrair seu relato da percepção do uso quanto a possíveis alterações no equilíbrio, postura corporal podendo interferir no desempenho esportivo. Este estudo é um ensaio clínico não randomizado que incluiu 65 atletas atendidos por demanda espontânea no ambulatório de Odontologia do Esporte da Faculdade de Odontologia da Universidade de São Paulo (FOUSP). A utilização do PBI teve influência significante na variável distancia glabela mento $(\mathrm{p}=0,005)$. 
Em relação ao relato da percepção de uso, 75,68\% relataram alterações no equilíbrio e 70,28\% na postura corporal, $70,28 \%$ relataram melhora de desempenho e $67,56 \%$ melhora no gesto esportivo. Conclui-se que utilização do PBI causa mais confiança nos atletas na execução dos exercícios e consequentemente, no desempenho esportivo.

Palavras-chave: Atletas; Protetores bucais; Equilíbrio postural; Fotogrametria.

\begin{abstract}
Mouthguards are intraoral devices that aim to prevent dental and maxillofacial trauma resulting from sports practice. As it is an interocclusal device, it is necessary to understand and know if it can interfere with the athlete's body balance, considering that the existence of the relationship between the temporomandibular system and postural control. The aim of this study was to evaluate the influence of individualized mouthguards on balance, head, jaw and shoulder posture of athletes and extract their account of the perception of use regarding possible changes in balance, body posture and sports performance. This study is a non-randomized clinical trial that included 65 athletes assisted by spontaneous demand at the Sports Dentistry Outpatient Clinic of the Dentistry School of the University of São Paulo (FOUSP). The use of individualized mouthguards had a significant influence on the variable glabellation distance $(\mathrm{p}=0.005)$. Regarding the report of the perception of use, $75.68 \%$ reported changes in balance and $70.28 \%$ in body posture, $70.28 \%$ reported improvement in performance and $67.56 \%$ improvement in sports gesture. It is concluded that the use of individualized mouthguards causes more confidence in athletes in the execution of exercises and, consequently, in sports performance.
\end{abstract}

Keywords: Athletes; Mouth protectors; Postural balance; Photogrammetry.

\title{
Resumen
}

Los protectores bucales son dispositivos intraorales que tienen como objetivo prevenir los traumatismos dentales y maxilofaciales resultantes de la práctica deportiva. Al tratarse de un dispositivo interoclusal, es necesario comprender y saber si puede interferir con el equilibrio corporal del deportista, considerando que la existencia de la relación entre el sistema temporomandibular y el control postural ya se ha comprobado mediante el análisis de diferentes oclusiones dentarias y posiciones mandibulares. El objetivo de este estudio fue evaluar la influencia de los protectores bucales individualizados en la postura y equilíbrio de los deportistas y extraer su relato de la percepción de uso con respecto a posibles cambios en el equilibrio, la postura corporal y el rendimiento deportivo. Este estudio es un ensayo clínico no aleatorizado que incluyó a 65 deportistas atendidos por demanda espontánea en la Clínica Ambulatoria de Odontología Deportiva de la Facultad de Odontología de la Universidad de São Paulo (FOUSP). El uso de los protectores bucales individualizados tuvo una influência significativa en la variable distancia de glabelación $(\mathrm{p}=$ 0,005). En cuanto al reporte de la percepción de uso, el 75,68\% reportó cambios en el equilibrio y el 70,28\% en la postura corporal, el 70,28\% reportó mejora en el rendimiento y el 67,56\% mejora en el gesto deportivo. Se concluye que el uso de los protectores bucales individualizados genera más confianza en los deportistas en la ejecución de los ejercicios y, en consecuencia, en el rendimiento deportivo.

Palabras clave: Atletas; Protectores bucales; Equilibrio postural; Fotogrametría.

\section{Introdução}

O controle postural é a relação estabelecida entre a postura e o equilíbrio corporal de modo que o indivíduo oriente-se e permaneça estável para correta execução das mecânicas corporais (Campelo, 2003; Saito et al., 2016). A coordenação do sistema de controle postural se dá pela interpretação das informações recebidas dos sistemas vestibular, visual e proprioceptivo, através do sistema nervoso central (SNC) (Baldaço et al., 2010; Freitas \& Barela, 2003; Souza, Barreiros, 2012).

O corpo humano representa um sistema articulado e interconectado. Sendo assim, mudanças em qualquer estrutura corporal podem levar a alterações músculo-articulares e ósseas, resultando em mecanismos de compensação, fato estes que podem interferir no gesto esportivo (Silvestrini-Biavati et al., 2013; Valentino \& Melito, 1991; Vieira Amantéa et al., 2004).

A associação entre o sistema estomatognático e a postura corporal já foi demonstrada na literatura (Cuccia \& Caradonna, 2009; Michelotti et al., 2011; Perinetti \& Contardo, 2009; Vieira Amantéa et al., 2004). O posicionamento da cabeça e do pescoço é mantido pelo equilíbrio, entre as estruturas das regiões cervical e oral, incluindo as estruturas ósseas, musculares e oclusão dentaria (Baldini et al., 2013). A existência da relação entre o sistema temporomandibular e o controle postural já foi comprovada analisando diferentes oclusões dentarias e posições mandibulares, aproximando a odontologia e a biomecânica (Scharnweber et al., 2017; Vieira Amantéa et al., 2004). 
Estudos avaliaram fatores que podem influenciar a relação controle postural realizados em placas estabilizadoras oclusais (Bolmont et al., 2002; Bracco et al., 2004; Lourenço \& Silva, 2013; Oliveira et al., 2019; Souza et al., 2006; Strini et al., 2009). Protetores bucais para esporte (PBI) são dispositivos intraorais que visam proteger dentes, lábio e língua e prevenindo traumatismos dentários e maxilofaciais decorrentes da prática esportiva (Padilha et al., 2021; Pae et al., 2013; Tuna \& Ozel, 2014; Vucic et al., 2016), tem suas limitações nas porções vestibular, palatina e oclusal. A hipótese desta pesquisa é que a exemplo das placas interoclusais o protetor bucal interfira de modo positivo na postura e equilíbrio do atleta. Sendo assim, o objetivo deste estudo foi observar, por meio de estabilometria e fotogrametria, se o protetor bucal individualizado para esporte (PBI) pode interferir no equilíbrio e postura corporal do atleta e sua percepção. Estas ações são propostas no intuito de conscientizar o atleta da necessidade do uso de proteção bucal durante a prática esportiva.

\section{Metodologia}

Este estudo é um ensaio clínico não randomizado que incluiu 65 atletas atendidos por demanda espontânea no ambulatório de Odontologia do Esporte da Faculdade de Odontologia da Universidade de São Paulo.

Foi confeccionado um PBI em Copolimero de Etileno e Acetato de Vinila (EVA) para cada participante. Foram formados 2 grupos distintos para 2 avaliações, fotogrametria (32 atletas) e estabilometria (33 atletas). Estas avaliações foram definidas seguindo protocolo de avaliação postural com fotogrametria de Figueiredo (2012) (Atkinson, 1996; Figueiredo et al., 2012) e o Protocolo de acordo com a Associação Francesa de Posturologia (Association Française de Posturologie, 1985; Gagey et al., 1984) conforme Figura 1.

Figura 1 - Distribuição dos indivíduos participantes nos grupos fotogrametria e estabilometria.

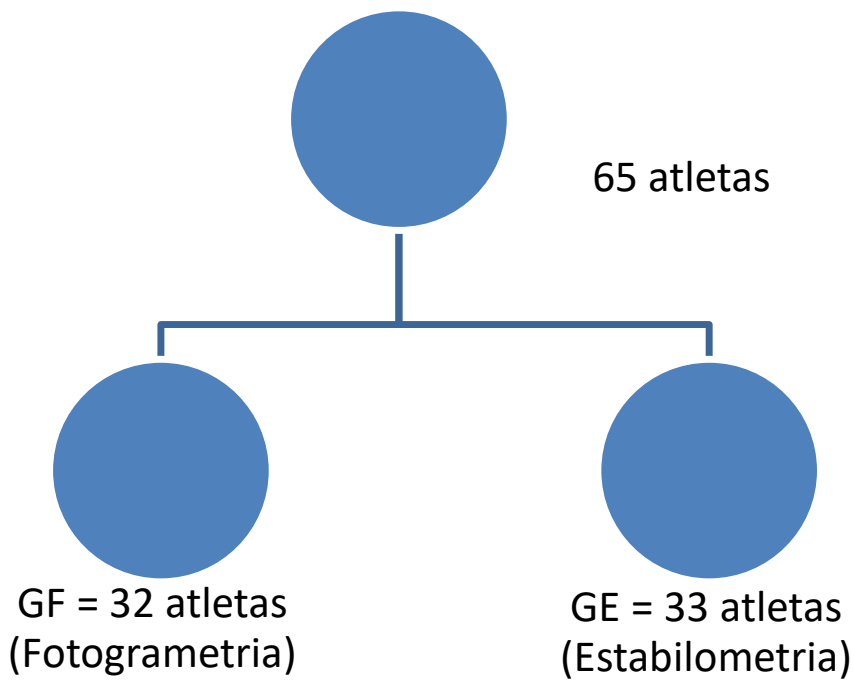

Fonte: Autores.

Optou-se por dividir o grupo de estudo em cada uma das técnicas, fotogrametria e estabilometria, para observar qual delas traduz melhor as alterações de equilíbrio e postura provocado por um dispositivo interoclusal com a intenção de proteção bucal, quando observados em uma população de atletas. Estas técnicas de avaliação foram selecionadas por serem comumente 
utilizadas em estudos de avaliação de dispositivos interoclusais, reabilitações e padrão oclusal associados a postura e equilíbrio. (Álvarez Solano et al., 2020; Candotti et al., 2019; Oliveira et al., 2019; Yoshida et al., 2008).

\section{Avaliação por Fotogrametria (GF)}

Para este teste foram realizadas tomadas fotográficas por um smartphone da marca Samsung® J5 Duos e com o auxílio de um tripé para padronização, praticidade e reprodutibilidade da distância e altura, conforme esquema demonstrado na Figura (Figura 2) (Figueiredo et al., 2012). Os indivíduos foram instruídos a permanecer em posição ortostática com a cabeça e olhar paralelos a linha de Frankfurt e em máxima intercuspidação. A posição dos pés foi determinada por um posicionador de forma a manter os calcanhares ligeiramente afastados e os pés abduzidos cerca de 15 graus. O tripé foi posicionado a 3 metros de distância do indivíduo e o smartphone a 1 metro de altura. Para calibração da imagem foi utilizado fio de prumo com dois marcadores de poliestireno distanciados 1 metro de acordo com o protocolo sugerido pelo Software de Avaliação Postural $\mathrm{SAPO}^{\circledR}$ (Ferreira et al., 2010). O atleta foi posicionado em frente a um simetrógrafo onde os pontos de interesse foram demarcados com esferas de poliestireno de $15 \mathrm{~mm}$ de diâmetro fixados com uma fita dupla face (Figura 3 A e 3B). Foram realizadas 3 tomadas fotográficas, vista anterior, sagital direita e esquerda.

Figura 2 - padronização da posição do atleta estudado para a avaliação por fotogrametria de acordo com o Software de Avaliação Postural SAPO ${ }^{\circledR}$.

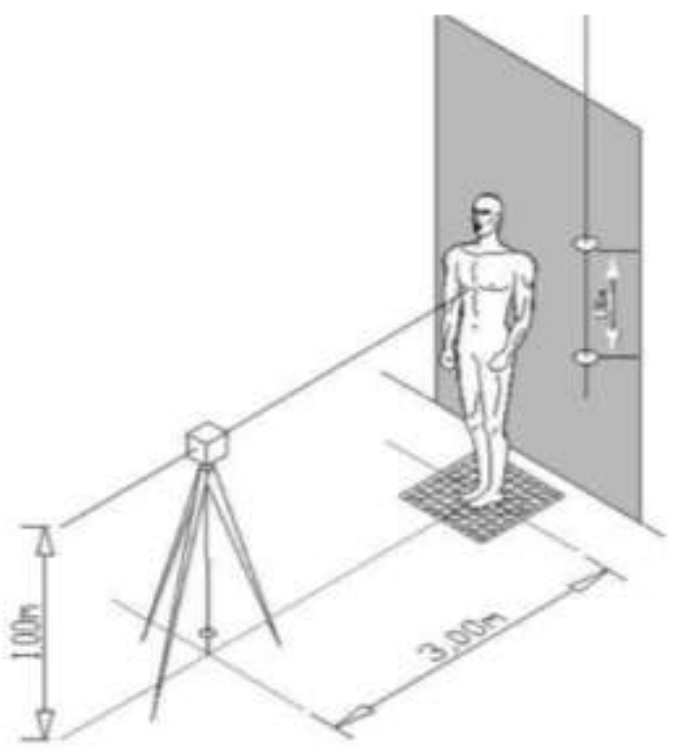

Fonte: Figueiredo et al. (2012).

Após a instalação do PBI com seus devidos ajustes, e o atleta realizou uma caminhada no local por 5 minutos com o intuito de estimular possíveis reorganizações corporais. Logo após foi novamente posicionado em frente ao simetrógrafo, onde foram realizadas mais 3 tomadas fotográficas nos mesmos critérios utilizados na primeira tomada. 
Figura 3 - Posicionamento do atleta em vista anterior e sagital com os pontos de demarcação nas regiões de interesse.
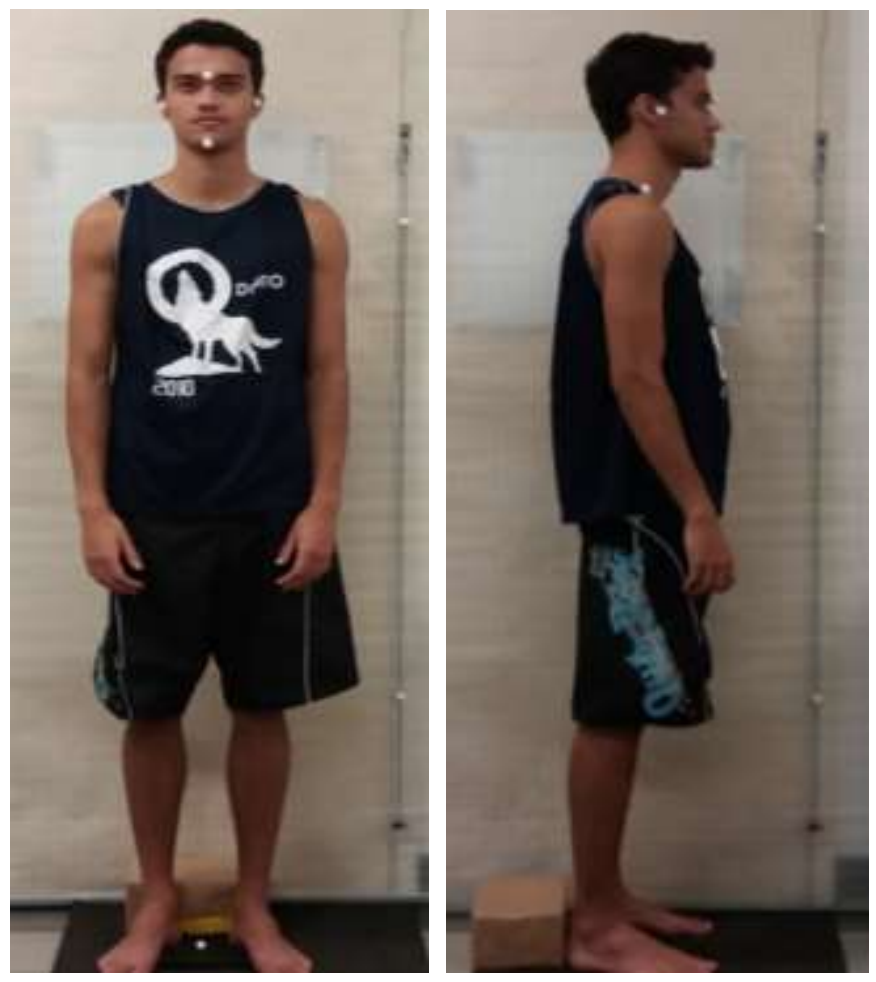

Fonte: Autores.

\section{Avaliação por Estabilometria (GE)}

Para este grupo foi realizada a avaliação pela plataforma de Baropodometria FootWork® (AM3, França. Versão 5.0.1.0). Este aparelho fornece informações estabilométricas relacionadas à base de suporte corporal em condições estática e dinâmica, permitindo análise do indivíduo a partir da área de superfície e amplitude do deslocamento do centro de pressão podal (CoP), tanto no sentido anteroposterior (CoP AP) como médio-lateral (CoP ML) como mostra a figura 4.

Cada atleta foi avaliado em 2 tempos de 20 s sem cortes, antes e após a instalação do PBI, utilizando posicionador podal, garantindo assim que todas as medidas seguissem o mesmo protocolo.

Figura 4 - Software Footwork e plataforma de baropodometria com posicionador de pés.

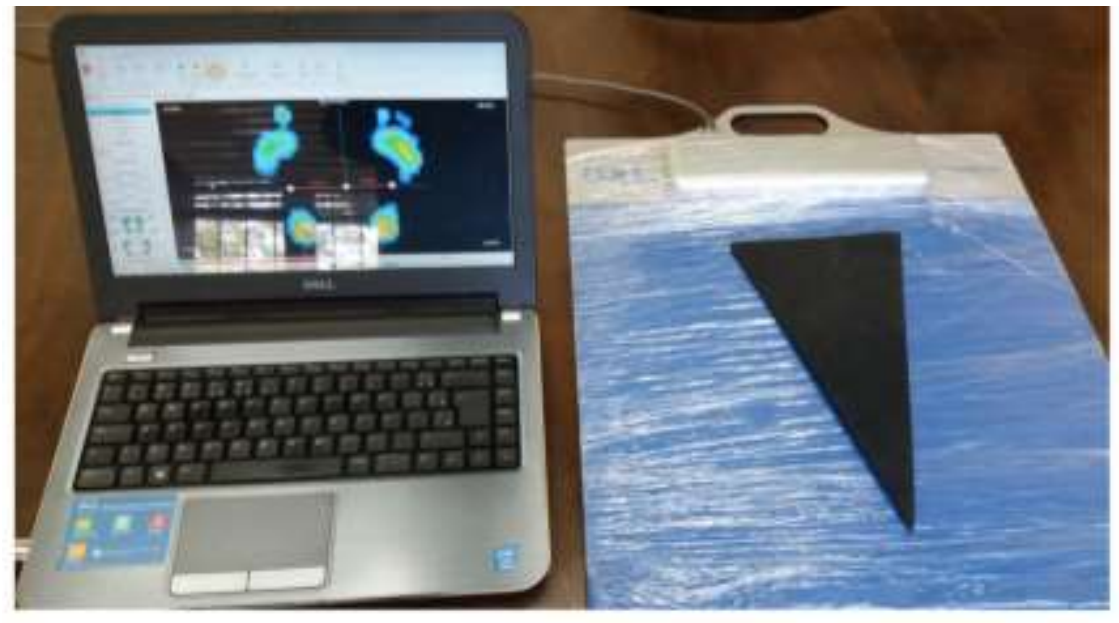

Fonte: Autores. 


\section{Avaliação Percepção individual da influência do PBI}

Vale ressaltar que após 6 a 12 meses de instalação dos PBIs e respectivas avaliações todos os 65 atletas foram convidados a responder um questionário sobre a utilização do PBI, no intuito de observar de modo subjetivo a percepção da postura e equilíbrio, segurança e desempenho esportivo no decorrer de seu uso, podendo ser uma métrica para observar a relevância clínica do incentivo ao uso do PBI durante a prática de esporte.

Esta pesquisa foi desenvolvida com aprovação do Comitê de Ética em Pesquisa sob o número de identificação: CAAE 69941217.2.0000.0075.

\section{Resultados}

A amostra para cada avaliação foi caracterizada conforme Tabela 1.

Tabela 1 - Característica da amostra.

\begin{tabular}{cccc}
\hline & Masculino & Feminino & $\begin{array}{c}\text { Média de } \\
\text { idade }\end{array}$ \\
\hline Fotogrametria & $46,87 \%$ & $53,13 \%$ & 22,24 anos \\
\hline Estabilometria & $81,81 \%$ & $18,19 \%$ & 23,09 anos \\
\hline Total & $64,61 \%$ & $35,39 \%$ & 22,67 anos \\
\hline
\end{tabular}

Fonte: Autores.

\section{Avaliação por Fotogrametria (GF)}

As seguintes análises foram nominadas e realizadas:

AHC - Alinhamento horizontal da cabeça: avaliado pelo ângulo formado entre os pontos tragus direito e tragus esquerdo;

AHA - Alinhamento horizontal dos acrômios: avaliado pelo ângulo formado entre os pontos acrômio direito e acrômio esquerdo;

rTA - Relação tragus: avaliado do ângulo formado entre as linhas tragus direito-esquerdo e acrômio direito-esquerdo;

dGM - Distância glabela-mento: avaliado pela distância entre os pontos glabela e mento;

AVC - Alinhamento vertical da cabeça: avaliado pelo ângulo formado entre os pontos tragus direito - acrômio direito e tragus direito - acrômio esquerdo, considerando a avaliação média entre os dois lados;

PM - Posição mandibular: avaliado por meio do ângulo formado entre a glabela e mento em vista sagital, quando valores negativos representaram retrusão e os positivos protrusão mandibular.

Deve-se observar que as medidas AHC e AVC foram consideradas para avaliação da posição da cabeça; dGM e PM para avaliação da posição mandibular; AHA para ombros e a medida rTA para avaliação da relação entre a postura da cabeça e ombros.

$\mathrm{Na}$ análise da fotogrametria as inclinações de cabeça detectadas para essas medidas no sentido horário (direita) receberam valor positivo, enquanto valores negativos representaram inclinações no sentido anti-horario (esquerda). 
Gráfico 1 - Apresentação do tamanho do efeito para grupo fotogrametria (GF).

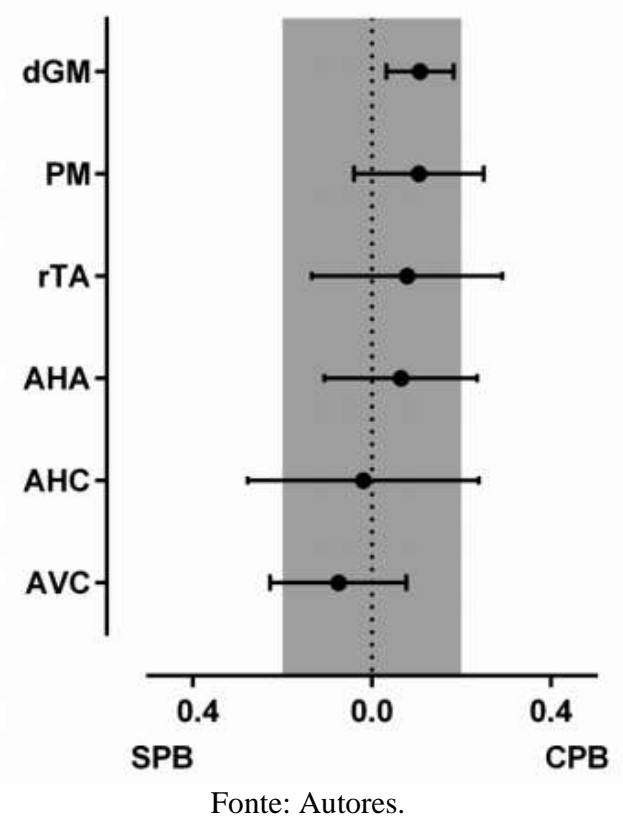

Observa-se no Gráfico 1 que a medida dGM apresentou resultados estatisticamente significante, efeito esperado já que o PBI tem influência na dimensão vertical de oclusão (DVO). O efeito clínico do uso do dispositivo foi considerado trivial em todas as variáveis de avaliação postural $(\mathrm{d}<0,2)$, possivelmente por ter sido registrado no dia da instalação do dispositivo.

A Tabela 2 mostra, para a observação antes (SPB) e depois (CPB) da instalação do PBI, a média, desvio padrão, intervalo de confiança (IC), análise "d de Cohen" e o valor de p obtido. A avaliação foi considerada significante quando o valor de $\mathrm{p} \leq 0,05$.

Tabela 2 - Avaliação postural por fotogrametria para $p \leq 0,05$.

\begin{tabular}{|c|c|c|c|c|c|c|}
\hline \multicolumn{2}{|c|}{ Fotogrametria } & \multirow{2}{*}{$\begin{array}{l}\text { Média } \\
-0,556\end{array}$} & \multirow{2}{*}{$\begin{array}{c}\begin{array}{c}\text { Desvio } \\
\text { Padrão }\end{array} \\
1,74\end{array}$} & \multirow{2}{*}{$\begin{array}{c}\text { IC } \\
0,60\end{array}$} & \multirow{3}{*}{$\begin{array}{c}\text { Cohen's d } \\
0,020\end{array}$} & \multirow{3}{*}{$\begin{array}{c}\begin{array}{c}\text { P- } \\
\text { valor }\end{array} \\
0,885\end{array}$} \\
\hline \multirow{2}{*}{$\mathrm{AHC}$} & SPB & & & & & \\
\hline & CPB & $-0,588$ & 1,37 & 0,47 & & \\
\hline \multirow{2}{*}{ AHA } & SPB & 0,159 & 1,55 & 0,54 & \multirow{2}{*}{0,066} & \multirow{2}{*}{0,463} \\
\hline & CPB & 0,266 & 1,69 & 0,58 & & \\
\hline \multirow{2}{*}{ rTA } & SPB & 1,841 & 1,61 & 0,56 & \multirow{2}{*}{0,080} & \multirow{2}{*}{0,472} \\
\hline & CPB & 1,966 & 1,55 & 0,53 & & \\
\hline \multirow{2}{*}{ AVC } & SPB & 13,32 & 5,6 & 1,95 & \multirow{2}{*}{0,076} & \multirow{2}{*}{0,339} \\
\hline & CPB & 12,89 & 5,71 & 1,98 & & \\
\hline \multirow{2}{*}{ PM } & SPB & 2,14 & 4,38 & 1,52 & \multirow{2}{*}{0,109} & \multirow{2}{*}{0,161} \\
\hline & CPB & 2,63 & 4,80 & 1,66 & & \\
\hline \multirow{2}{*}{ dGM } & SPB & 14,08 & 2,19 & 0,76 & \multirow{2}{*}{0,110} & \multirow{2}{*}{0,005} \\
\hline & CPB & 14,31 & 2,12 & 0,74 & & \\
\hline
\end{tabular}

Fonte: Autores. 


\section{Avaliação por Estabilometria (GE)}

$\mathrm{Na}$ avaliação por estabilometria observou-se o centro de pressão podal (CoP), no sentido médio-lateral (CoP ML) e no sentido anteroposterior (CoP AP).

O Gráfico 2 e a Tabela 3 mostram para a observação antes (SPB) e depois (CPB) da instalação do PBI, a média, desvio padrão, intervalo de confiança (IC), análise "d de Cohen" e o valor de p obtido mostram os dados da avaliação, considerando o efeito significante quando $\mathrm{p} \leq 0,05$.

Gráfico 2 - Apresentação do tamanho do efeito para o grupo estabilometria (GE).

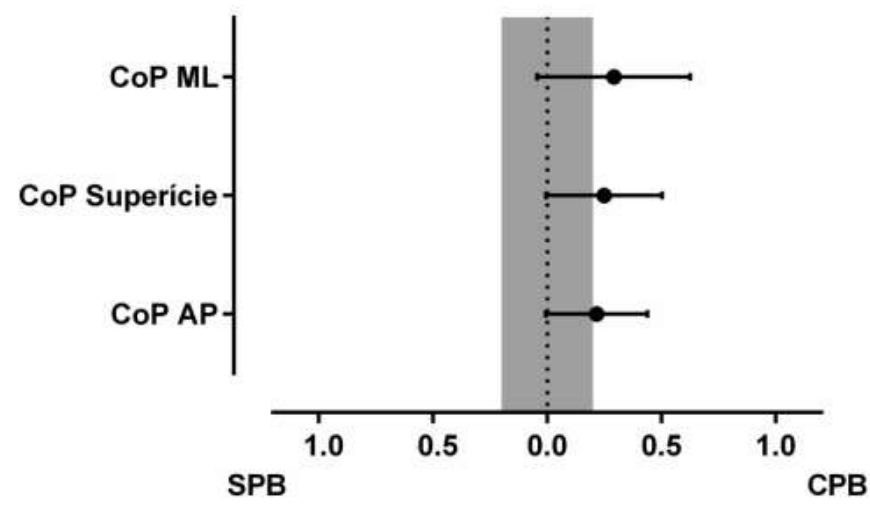

Fonte: Autores.

Tabela 3 - Avaliação do equilíbrio por estabilometria para $p \leq 0,05$.

\begin{tabular}{|c|c|c|c|c|c|c|}
\hline \multicolumn{2}{|c|}{ Estabilometria } & \multirow{2}{*}{$\begin{array}{l}\text { Média } \\
0,952\end{array}$} & \multirow{2}{*}{$\begin{array}{c}\text { Desvio } \\
\text { Padrão } \\
0,44\end{array}$} & \multirow{2}{*}{$\begin{array}{c}\text { IC } \\
0,15\end{array}$} & \multirow{2}{*}{$\begin{array}{c}\text { Cohen's } \\
\text { d }\end{array}$} & \multirow[t]{2}{*}{ P-valor } \\
\hline & SPB & & & & & \\
\hline \multirow[t]{3}{*}{ CoP ML } & & & & & 0,292 & 0,091 \\
\hline & CPB & 1,119 & 0,61 & 0,21 & & \\
\hline & SPB & 1,501 & 0,80 & 0,27 & & \\
\hline \multirow[t]{2}{*}{ CoP AP } & & & & & 0,216 & 0,059 \\
\hline & CPB & 1,790 & 1,32 & 0,46 & & \\
\hline \multirow{3}{*}{$\begin{array}{l}\text { Superficie } \\
\text { COP }\end{array}$} & SPB & 1,399 & 1,98 & 0,68 & \multirow{3}{*}{0,249} & \multirow{3}{*}{0,057} \\
\hline & & & & & & \\
\hline & CPB & 1,965 & 2,35 & 0,81 & & \\
\hline
\end{tabular}

Fonte: Autores.

\section{Avaliação Percepção individual da influência do PBI}

Dos 65 atletas que passaram pelas avaliações, 52,85\% responderam ao questionário. A Tabela 4 mostra de modo dicotômico a análise descritiva realizada na avaliação da percepção da influência do PBI. 
Tabela 4 - Avaliação da percepção da influência do PBI, realizada seis meses após a instalação do PBI.

\begin{tabular}{l|c|c}
\hline & Sim & Não \\
\hline $\begin{array}{l}\text { Você sente mais confiança para treinar e } \\
\text { competir com uso de PBI? }\end{array}$ & $97,29 \%$ & $2,71 \%$ \\
\hline $\begin{array}{l}\text { Você sente maior facilidade para executar } \\
\text { gestos esportivos com uso de PBI? }\end{array}$ & $67,56 \%$ & $32,44 \%$ \\
\hline $\begin{array}{l}\text { Você sente melhora no seu equilibrio corporal } \\
\text { com uso de PBI? }\end{array}$ & $24,32 \%$ & $75,68 \%$ \\
\hline $\begin{array}{l}\text { Você sente alguma mudança postural com uso } \\
\text { de PBI? }\end{array}$ & $29,72 \%$ & $70,28 \%$ \\
\hline $\begin{array}{l}\text { Você sente melhora do seu desempenho como } \\
\text { atleta com uso de PBI? }\end{array}$ & $70,28 \%$ & $29,72 \%$ \\
\hline $\begin{array}{l}\text { Você sente piora, seja do seu equilíbrio, } \\
\text { postura e/ou desempenho com uso de PBI? }\end{array}$ & $2,71 \%$ & $97,3 \%$ \\
\hline
\end{tabular}

Fonte: Autores.

\section{Discussão}

Este estudo buscou avaliar a influência da presença de protetor bucal para esporte na postura e equilíbrio do atleta por meio de estabilometria e fotogrametria. O interesse por esta pesquisa surgiu da observação de trabalhos científicos que mostram que placas interoclusais podem interferir na postura do paciente (Maurer et al., 2018; Scharnweber et al., 2017; Vieira Amantéa et al., 2004). A interferência benéfica do protetor bucal para esporte na postura e equilíbrio do atleta seria muito interessante, estimulando o atleta a usar tal dispositivo, não só objetivando a proteção orofacial mas também de ajustar sua postura e garantir seu equilíbrio, interferindo assim em seu desempenho esportivo.

Os resultados mostraram que no sentido vertical houve abaixamento da mandíbula representado pelo aumento da dGM por consequência do aumento da dimensão vertical de oclusão (DVO), ou seja, da distância entre as superfícies oclusais. Essa alteração, no entanto, não teve efeito clínico considerado importante. Porém alguns autores afirmaram que aumento na DVO é capaz de alterar a posição da cabeça e coluna vertebral (Andrighetto \& de Fantini, 2015; Ohlendorf et al., 2014).

Este estudo não encontrou alterações do posicionamento da cabeça, sagital da mandíbula e ombros (AHC, AVC, PM, rTA, AHA). As medições foram tomadas logo após (5 minutos) a instalação do PBI, podendo justificar a ausência de alterações neste posicionamento por conta do curto período de reavaliação.

Quando existe desequilíbrio da atividade muscular mastigatória ocorrem alterações da musculatura cervical e dos ombros (Vieira Amantéa et al., 2004). A realização do ajuste oclusal do PBI evitando contatos prematuros e desequilíbrio muscular mastigatório, bem como o tempo de uso do dispositivo podem justificar a ausência de alterações no AHA e rTA entre as condições sem e com PBI (Moya et al., 1994).

Sabe-se que em momentos de esforço os atletas demonstram tensão, apertamento dentário e contração da musculatura facial e do pescoço gerando estresse ao sistema craniomandibular (Garabee, 1981). As fibras do músculo temporal anterior correm quase perpendiculares ao plano oclusal, o que as torna particularmente responsivas a mudanças na dimensão vertical 
(Al Quran \& Lyons, 1999). O músculo masseter superficial corre em um ângulo ligeiramente oblíquo de sua fixação na borda inferior com o zigoma, que ajuda a estabilizar a relação maxilomandibular durante movimentos atléticos explosivos (Gage et al., 2015).

Em 2013, Baldini et al., demostrou que indivíduos com Desordem Temporomandibular miógena apresentaram diferença significativamente na estabilidade postural corporal através da avaliação por estabilometria, comparado a pacientes saudáveis. Indicando área de oscilação e velocidade de oscilação aumentados nestes indivíduos, fatores estes que poderiam afetar o desempenho esportivo de atletas. Em nosso estudo, esperava-se que um aumento no DVO causado pela utilização do PBI, possivelmente indicaria melhora postural dessas variáveis também (Baldini et al., 2013).

Em um estudo realizado com jogadores de basquete em 2020 por Nam et al., mostrou que atletas que utilizaram o PBI por um tempo prolongado (8 semanas) tiveram um aumento significativo no ângulo cifótico comparado com atletas que não usam o PBI, ou usam por pouco tempo (7 dias). Ele também relatou efeito significativo no desempenho do equilíbrio, tanto estático, quanto dinâmico para a utilização de 8 semanas, promovendo assim um benefício no desempenho. Este estudo nos encoraja a realizar um novo estudo com um período de acompanhamento maior dos atletas e a utilização de outras tecnologias para avaliação (Nam et al., 2020).

De acordo com Pae et al., 2013 o uso de protetores bucais quando ajustados bilateralmente equilibrando a oclusão permitiram movimento mais acelerado do taco de golfe, porém não causou alteração da velocidade da bola e da precisão da jogada. Receptores periféricos na ATM, músculos, ligamentos periodontais e tecidos moles (língua, lábios e a mucosa oral) notam a presença do PBI e do aumento da DVO, causando diminuição da atividade muscular mastigatória (Al Quran \& Lyons, 1999; Golem \& Arent, 2015; Pae et al., 2013). Raquel et al., 2017 afirmou que o uso do PBI evita que os músculos mastigatórios sejam submetidos a estresse repetitivo que podem desequilibrar a musculatura orofacial, prejudicando assim o atleta. Dessa forma, o aumento da DVO parece afetar a ativação muscular e desempenho no resto do corpo (Gelb et al., 1996; Raquel et al., 2017).

Um estudo realizado em atletas de karatê avaliou o uso dos protetores bucais individualizados e foi possível observar que o uso de protetores bucais individualizados proporcionou melhor estabilidade dos músculos mastigatórios durante o treinamento (Raquel et al., 2017).

Acredita-se que o posicionamento ideal da articulação temporomandibular é capaz de melhorar o posicionamento das vértebras cervicais e promover sinalização neural afetando o desempenho muscular de outras partes do corpo (Gage et al., 2015). Os sensores somatossensoriais são importantes para detecção, orientação e posicionamento dos seguimentos corporais para manutenção do equilíbrio (Freitas \& Barela, 2003). Segundo Sforza et al., o posicionamento simétrico da mandíbula resulta em contrações musculares simétricas e redução da oscilação do corpo (Sforza et al., 2006).

Valores da área de oscilação do CoP aumentados podem sugerir diminuição do controle postural ou apenas que é necessário maior deslocamento do CoP para manter a estabilidade sem necessariamente afetar negativamente o equilíbrio do indivíduo (Ingersoll \& Armstrong, 1992; Palmieri et al., 2002).

Scharnweber et al., (2017) afirma que o bloqueio de intercuspidação leva a redução do deslocamento de CoP, enquanto Baldini et al., (2013) afirmou existir uma fraca correlação entre o aumento da DVO e área de deslocamento e oscilação do CoP. Outros autores não encontraram influência de diferentes posturas da mandíbula em relação ao deslocamento do CoP (Baldini et al., 2013; Ferrario et al., 1996; Michelotti et al., 2011; Scharnweber et al., 2017). Bracco et al., (2004) apresentaram resultados significantes no deslocamento de CoP em diferentes posturas mandibulares. Gangloff et al., (2000) relatou diminuição dos deslocamentos de CoP com o aumento da DVO por uso de placa de mordida. Dunn-Lewis et al., (2012) e Golem \& Arent (2015) mostraram que o uso do PBI não altera o equilíbrio dinâmico do indivíduo (Bracco et al., 2004; Dunn-Lewis et al., 2012; Gangloff et al., 2000; Golem \& Arent, 2015). A literatura sugere que alterações posturais pelo o 
aumento de DVO podem não ocorrer em indivíduos que não possuam disfunções posturais e também que pode haver impedimento da sobrecarga na articulação e, por isso, diminuição da sinalização neurológica do sistema estomatognático (Al Quran \& Lyons, 1999; Moya et al., 1994). É possível também que intervenções odontológicas que alteram o posicionamento mandibular não manifestem alterações por causarem pouca diferença na aferência trigeminal e não influenciarem no sistema de controle postural, causando compensação e mudança no equilíbrio e postura corporal (Nanda et al., 2011).

Este estudo verificou a percepção positiva dos indivíduos em relação ao equilíbrio, postura e desempenho esportivo dos atletas ao utilizaram o PBI. Bolmont et al., (2002) mostrou que estados de humor e ansiedade podem afetar o equilíbrio do indivíduo, aumentar a disposição física e compensar efeitos adversos da ansiedade. Corroborando com Gage et al., a maioria relatou efeitos positivos no desempenho físico ao utilizarem o dispositivo (Bolmont et al., 2002; Gage et al., 2015). Os efeitos na postura e equilíbrio foram pouco relatados (70,28\% e 75,68\%, respectivamente).

Autores avaliaram o efeito no PBI no sistema de controle postural. Mizumachi et al., (2008) verificaram os efeitos do PB no sistema vestibular e não apresentou resultados significantes quanto à sua influência no reflexo vestíbulo-cólico que controla a musculatura do pescoço (Mizumachi et al., 2008; Ricotti, 2011). Este estudo procurou apresentar a influência do PBI no resultado das informações recebidas pelo SNC por meio do sistema somatossensorial não obtendo resultados significantes ou relevantes clinicamente.

Como o PBI é um dispositivo interoclusal, a ideia de avaliar os efeitos do seu uso nesses quesitos surgiu da indicação e da necessidade do incentivo à prevenção de traumatismo orofacial durante a prática esportiva. Conhecer se os dispositivos de proteção indicados podem influenciar no dia-a-dia dos atletas que o utilizam é fundamental para que mais indivíduos não negligenciem sua necessidade.

Sugere-se, portanto, que novos estudos sejam realizados a fim de analisar se o PBI influencia partes específicas do sistema de controle postural. Propõe-se também fazer os estudos realizados neste trabalho no mesmo grupo de atletas e promover registros mensais ou trimestrais, por um período maior, com medições dinâmicas, com olhos fechados e abertos para ampliar a observação da interferência do PBI na postura e equilíbrio corporal.

\section{Conclusão}

O uso do PBI não interfere na postura da cabeça, mandíbula e ombros de atletas quando observado logo após sua instalação.

Os atletas relataram sentir mais confiança e observaram melhor desempenho esportivo durante a utilização do PBI mostrando interesse por seu uso.

\section{Referências}

Al Quran, F. A. M., \& Lyons, M. F. (1999). The immediate effect of hard and soft splints on the EMG activity of the masseter and temporalis muscles. Journal of Oral Rehabilitation, 26(7), 559-563. https://doi.org/10.1046/j.1365-2842.1999.00421.x

Álvarez Solano, C., González Camacho, L. A., Castaño Duque, S. P., Cortés Velosa, T., Vanoy Martin, J. A., \& Chambrone, L. (2020). To evaluate whether there is a relationship between occlusion and body posture as delineated by a stabilometric platform: A systematic review. CRANIO®, $1-12$.

Andrighetto, A. R., \& de Fantini, S. M. (2015). Effects of neuromuscular deprogramming on the head position. Cranio - Journal of Craniomandibular Practice, 33(3), 183-188. https://doi.org/10.1179/2151090314Y.0000000016

Association Française de Posturologie. (1985). Normes 85. Editées Par I'ADAP (Association Pour Le Développment et l'Application de La Posturologie) 20.

Atkinson, K. B. (1996). Close range photogrammetry and machine vision. Whittles Publ.

Baldaço, F. O., Cadó, V. P., de Souza, J., Mota, C. B., \& Lemos, J. C. (2010). Análise do treinamento proprioceptivo no equilíbrio de atletas de futsal feminino TÍTULO Analysis of proprioceptive training in the balance of women's futsal athletes. Fisioterapia Em Movimento, 23(2), $183-192$.

Baldini, A., Nota, A., Tripodi, D., Longoni, S., \& Cozza, P. (2013). Evaluation of the correlation between dental occlusion and posture using a force platform. Clinics, 68(1), 45-49. https://doi.org/10.6061/clinics/2013(01)OA07 
Bolmont, B., Gangloff, P., Vouriot, A., \& Perrin, P. P. (2002). Mood states and anxiety influence abilities to maintain balance control in healthy human subjects. Neuroscience Letters, 329(1), 96-100. https://doi.org/10.1016/S0304-3940(02)00578-5

Bracco, P., Deregibus, A., \& Piscetta, R. (2004). Effects of different jaw relations on postural stability in human subjects. Neuroscience Letters, 356(3), 228230. https://doi.org/10.1016/j.neulet.2003.11.055

Campelo, T. de S. (2003). Postura e equilíbrio corporal: estudo das relações existentes. 44.

Candotti, C. T., Gelain, G. M., Antoniolli, A., Araújo, L. M., Vieira, A., \& Loss, J. F. (2019). Repeatability and Reproducibility of Postural Variables by Photogrammetry. Journal of Manipulative and Physiological Therapeutics, 42(5), 372-378. https://doi.org/10.1016/j.jmpt.2018.10.006

Cuccia, A., \& Caradonna, C. (2009). The relationship between the stomatognathic system and body posture. Clinics, 64(1), 61-66. https://doi.org/10.1590/S1807-59322009000100011

Dunn-Lewis, C., Luk, H.-Y., Comstock, B. A., Szivak, T. K., Hooper, D. R., Kupchak, B. R., Watts, A. M., Putney, B. J., Hydren, J. R., \& Volek, J. S. (2012). The effects of a customized over-the-counter mouth guard on neuromuscular force and power production in trained men and women. The Journal of Strength \& Conditioning Research, 26(4), 1085-1093.

Ferrario, V. F., Sforza, C., Schmitz, J. H., \& Taroni, A. (1996). Occlusion and center of foot pressure variation: is there a relationship? The Journal of Prosthetic Dentistry, 76(3), 302-308.

Ferreira, E. A. G., Duarte, M., Maldonado, E. P., Burke, T. N., \& Marques, A. P. (2010). Postural assessment software (PAS/SAPO): Validation and reliabiliy. Clinics, 65(7), 675-681. https://doi.org/10.1590/S1807-59322010000700005

Figueiredo, R. V., Amaral, A. C., \& Shimano, A. C. (2012). Photogrammetry on the identifi cation of postural asymmetries in cadets and pilots of the Brazilian air force academy. Brazilian Journal of Physical Therapy, 16(1), 54-60. https://doi.org/10.1590/S1413-35552012000100010

Freitas, P., \& Barela, J. (2003). Características Comportamentais Do Controle Postural De Jovens , Adultos E Idosos. Thesis, 130.

Gage, C. C., Huxel Bliven, K. C., Bay, R. C., Sturgill, J. S., \& Park, J. H. (2015). Effects of mouthguards on vertical dimen sion, muscle activation, and athlete preference: a prospective cross-sectional study. Gen Dent, 63(6), 48-55.

Gagey, P. M., Gentaz, R., Guillamon, J. L., Bizzo, G., Bregeard, C., Debruille, C., \& Baudry, L. (1984). Études statistiques des mesures faites sur l’homme normal à l'aide de la plate-forme de stabilométrie clinique normalisée. Agressologie.

Gangloff, P., Louis, J.-P., \& Perrin, P. P. (2000). Dental occlusion modifies gaze and posture stabilization in human subjects. Neuroscience Letters, 293(3), 203-206.

Garabee, W. F. (1981). Performance in the Long Distance Runner - A Three Year Study.pdf. Craniomandibular Orthopedics and Athletic, 4(3).

Gelb, H., Mehta, N. R., \& Forgione, A. G. (1996). The relationship between jaw posture and muscular strength in sports dentistry: A reappraisal. Cranio, 14(4), 320-325. https://doi.org/10.1080/08869634.1996.11745984

Golem, D. L., \& Arent, S. M. (2015). Effects of over-the-counter jaw-repositioning mouth guards on dynamic balance, flexibility, agility, strength, and power in college-aged male athletes. The Journal of Strength \& Conditioning Research, 29(2), 500-512.

Ingersoll, C. D., \& Armstrong, C. W. (1992). The effects of closed-head injury on postural sway. Medicine and Science in Sports and Exercise, 24(7), 739743 .

Lourenço, C. P., \& Silva, A. L. dos S. (2013). Posture control and vestibular oculomotor system in pistol sport shooters. Posture Control and Vestibular Oculomotor System in Pistol Sport Shooters, 19(5), 313-316. https://doi.org/10.1590/S1517-86922013000500002

Maurer, C., Heller, S., Sure, J. J., Fuchs, D., Mickel, C., Wanke, E. M., Groneberg, D. A., \& Ohlendorf, D. (2018). Strength improvements through occlusal splints? The effects of different lower jaw positions on maximal isometric force production and performance in different jumping types. PLoS ONE, 13(2), 117. https://doi.org/10.1371/journal.pone.0193540

Michelotti, A., Buonocore, G., Manzo, P., Pellegrino, G., \& Farella, M. (2011). Dental occlusion and posture: An overview. Progress in Orthodontics, 12(1), 53-58. https://doi.org/10.1016/j.pio.2010.09.010

Mizumachi, M., Sumita, Y., \& Ueno, T. (2008). Effect of wearing a mouthguard on the vestibulocollic reflex. Journal of Science and Medicine in Sport, 11(2), $191-197$.

Moya, H., Miralles, R., Zuñiga, C., Carvajal, R., Rocabado, M., \& Santander, H. (1994). Influence of stabilization occlusal splint on craniocervical relationships. Part I: Cephalometric analysis. CRANIO®, 12(1), 47-51.

Nam, H. J., Lee, J. H., Hong, D. S., \& Jung, H. C. (2020). The effect of wearing a customized mouthguard on body alignment and balance performance in professional basketball players. International Journal of Environmental Research and Public Health, 17(17), 1-9. https://doi.org/10.3390/ijerph17176431

Nanda, A., Jain, V., \& Srivastava, A. (2011). An electromyographic study to assess the minimal time duration for using the splint to raise the vertical dimension in patients with generalized attrition of teeth. Indian Journal of Dental Research, 22(2).

Ohlendorf, D., Seebach, K., Hoerzer, S., Nigg, S., \& Kopp, S. (2014). The effects of a temporarily manipulated dental occlusion on the position of the spine: A comparison during standing and walking. Spine Journal, 14(10), 2384-2391. https://doi.org/10.1016/j.spinee.2014.01.045 
Oliveira, S. S. I., Pannuti, C. M., Paranhos, K. S., Tanganeli, J. P. C., Laganá, D. C., Sesma, N., Duarte, M., Frigerio, M. L. M. A., \& Cho, S. C. (2019). Effect of occlusal splint and therapeutic exercises on postural balance of patients with signs and symptoms of temporomandibular disorder. Clinical and Experimental Dental Research, 5(2), 109-115. https://doi.org/10.1002/cre2.136

Padilha, A. C. L., Constante, H. M., Fronza, H. P., \& Coto, N. P. (2021). Orofacial trauma and mouthguard use in Brazilian rugby union players. Dental Traumatology, 37(1), 53-57. https://doi.org/10.1111/edt.12592

Pae, A., Yoo, R. K., Noh, K., Paek, J., \& Kwon, K. R. (2013). The effects of mouthguards on the athletic ability of professional golfers. Dental Traumatology, 29(1), 47-51. https://doi.org/10.1111/j.1600-9657.2012.01123.x

Palmieri, R. M., Ingersoll, C. D., Stone, M. B., \& Krause, B. A. (2002). Center-of-pressure parameters used in the assessment of postural control. Journal of Sport Rehabilitation, 11(1), 51-66.

Perinetti, G., \& Contardo, L. (2009). Posturography as a diagnostic aid in dentistry: A systematic review. Journal of Oral Rehabilitation, 36(12), 922-936. https://doi.org/10.1111/j.1365-2842.2009.02019.x

Raquel, G., Namba, E. L., Bonotto, D., Rosa, E. A. R., Trevilatto, P. C., Machado, M. Â. N., Vianna-Lara, M. S., \& Azevedo-Alanis, L. R. (2017). The use of a custom-made mouthguard stabilizes the electromyographic activity of the masticatory muscles among Karate-Dō athletes. Journal of Bodywork and Movement Therapies, 21(1), 109-116.

Ricotti, L. (2011). Static and dynamic balance in young athletes. Journal of Human Sport and Exercise, 6(4), 616-628.

Saito, A. K., Navarro, M., Silva, M. F., Arie, E. K., \& Peccin, M. S. (2016). Oscilação do centro de pressão plantar de atletas e não atletas com e sem entorse de tornozelo. Revista Brasileira de Ortopedia, 51(4), 437-443. https://doi.org/10.1016/j.rbo.2015.10.001

Scharnweber, B., Adjami, F., Schuster, G., Kopp, S., Natrup, J., Erbe, C., \& Ohlendorf, D. (2017). Influence of dental occlusion on postural control and plantar pressure distribution. Cranio - Journal of Craniomandibular Practice, 35(6), 358-366. https://doi.org/10.1080/08869634.2016.1244971

Sforza, C., Tartaglia, G. M., Solimene, U., Morgun, V., Kaspranskiy, R. R., \& Ferrario, V. F. (2006). Occlusion, sternocleidomastoid muscle activity, and body sway: a pilot study in male astronauts. CRANIO®, 24(1), 43-49.

Silvestrini-Biavati, A., Migliorati, M., Demarziani, E., Tecco, S., Silvestrini-Biavati, P., Polimeni, A., \& Saccucci, M. (2013). Clinical association between teeth malocclusions, Wrong posture and ocular convergence disorders: An epidemiological investigation on primary school children. BMC Pediatrics, 13(1). https://doi.org/10.1186/1471-2431-13-12

Souza, G. S. de, Gonçalves, D. F., \& Pastre, C. M. (2006). Propriocepção cervical e equilíbrio: uma revisão. Fisioter. Mov, 19(4), 33-40.

Souza, L. A., Barreiros, S. (2012). Avaliação da Postura e Desempenho dos jogadores seniores do Industrial Desportivo Vieirense.

Strini, P. J. S. A., Machado, N. A. de G., Gorreri, M. C., Ferreira, A. de F., Sousa, G. da C., \& Fernandes Neto, A. J. (2009). Postural evaluation of patients with temporomandibular disorders under use of occlusal splints. Journal of Applied Oral Science, 17(5), 539-543. https://doi.org/10.1590/S167877572009000500033

Tuna, E. B., \& Ozel, E. (2014). Factors affecting sports-related orofacial injuries and the importance of mouthguards. Sports Medicine, 44(6), 777-783. https://doi.org/10.1007/s40279-014-0167-9

Valentino, B., \& Melito, F. (1991). Valentino and Melito 1991 - Functional Relationships Between the Muscles of Matication and the Muscles of the Leg. 3337 .

Vieira Amantéa, D., Novaes, A. P., Campolongo, G. D., Pessoa, T., \& Barros, D. (2004). The importance of the postural evaluation in patients with temporomandibular joint dysfunction. Acta Ortopédica Brasileira, 12(3), 155-159.

Vucic, S., Drost, R. W., Ongkosuwito, E. M., \& Wolvius, E. B. (2016). Dentofacial trauma and players' attitude towards mouthguard use in field hockey: A systematic review and meta-analysis. British Journal of Sports Medicine, 50(5), 298-304. https://doi.org/10.1136/bjsports-2015-094896

Yoshida, M., Kikutani, T., Okada, G., Kawamura, T., Kimura, M., \& Akagawa, Y. (2008). The effect of tooth loss on body balance control among community-dwelling elderly persons. The International Journal of Prosthodontics, 22(2), 136-139. http://www.ncbi.nlm.nih.gov/pubmed/19418857 